\title{
Topical Glycopyrronium Tosylate in Primary Axillary Hyperhidrosis: A Profile of Its Use
}

\author{
Yvette N. Lamb ${ }^{1}$
}

Published online: 30 September 2019

() Springer Nature 2019, corrected publication 2019

\begin{abstract}
Glycopyrronium tosylate $\left(\mathrm{Qbrexza}^{\mathrm{TM}}\right)$ is available as single-use, pre-moistened cloths and has been approved in the USA for the topical treatment of primary axillary hyperhidrosis in adults and children $\geq 9$ years of age. Glycopyrronium tosylate is effective in reducing patient-reported severity of disease and gravimetrically measured sweat production in this patient population; improvements have been shown to be maintained throughout long-term treatment (up to 48 weeks). Glycopyrronium tosylate is generally well tolerated, with most adverse events being mild to moderate in severity. Glycopyrronium tosylate thus provides a self-administered, non-invasive alternative to topical antiperspirant therapy and clinic-based treatments in adults with primary axillary hyperhidrosis, and is the only alternative to topical antiperspirants specifically approved in children and adolescents $\geq 9$ years of age.
\end{abstract}

Adis evaluation of topical glycopyrronium tosylate in primary axillary hyperhidrosis

First US-approved topical anticholinergic treatment for this indication; applied once daily with a single-use cloth

Suitable for use in patients $\geq 9$ years of age

Effective in improving disease severity, sweat production and quality of life

Generally well tolerated, with adverse events infrequently leading to treatment discontinuation
Enhanced material for this Adis Drug Q\&A can be found at https ://doi.org/10.6084/m9.figshare.9789284.

Yvette N. Lamb

demail@springer.com

1 Springer Nature, Private Bag 65901, Mairangi Bay, Auckland 0754, New Zealand

\section{What is the Rationale for Developing Topical Glycopyrronium Tosylate in Primary Axillary Hyperhidrosis?}

Hyperhidrosis is a skin condition characterised by sweat production in excess of that which is physiologically necessary for normal thermoregulation [1-4]. Hyperhidrosis can be classified as either primary (i.e. idiopathic) or secondary to a medication or underlying medical condition. In primary hyperhidrosis, which is more common, excessive sweating is typically bilaterally symmetrical and localized; the sites most frequently affected are the axillae (underarms), palms, soles or craniofacial regions [1-4]. While the exact cause is not yet understood, primary hyperhidrosis is thought to be the result of autonomic nervous system dysregulation leading to the overstimulation of cholinergic receptors on the eccrine sweat glands [3,5], which are present in high densities in the commonly affected areas [3]. In the USA, the estimated prevalence of hyperhidrosis is $4.8 \%(\approx 15.3$ million people) and is highest in young adults aged 18-39 years [1]; onset of primary hyperhidrosis is generally at or prior to the age of 25 [4].

Hyperhidrosis varies in severity and can have a considerable impact on quality of life, with $70 \%$ of patients with hyperhidrosis reporting severe sweating that is barely tolerable or intolerable and frequently or always interfering with their daily activities [1]. This interference can extend into the emotional, social, professional and educational spheres 
of their lives $[2,6]$, and patients with hyperhidrosis report significantly higher rates of anxiety and depression than those without [7].
While patients with hyperhidrosis often fail to seek the advice of healthcare professionals due to a lack of awareness that they have a treatable medical condition $[1,8]$, the early

Table 1 Summary of the prescribing information of glycopyrronium tosylate topical formulation (Qbrexza ${ }^{\mathrm{TM}}$ ) in primary axillary hyperhidrosis in the USA [11]

What is the approved indication for glycopyrronium tosylate?

The topical treatment of primary axillary hyperhidrosis in adults and pediatric pts $\geq 9$ years of age

How is glycopyrronium tosylate available?

Single-use cloths pre-moistened with $2.4 \%$ glycopyrronium solution (packaged in individual pouches)

How should glycopyrronium tosylate be applied?

For topical use in underarm area only; do not use in other areas of body

Apply to clean, dry skin; do not apply to broken skin and avoid using with occlusive dressings

Remove cloth from pouch and unfold, wipe across one entire underarm once and then wipe the same cloth across the other underarm once After applying, discard cloth and immediately wash hands with soap and water

Avoid transferring glycopyrronium tosylate to the periocular area; if glycopyrronium tosylate comes into contact with eyes, it may cause temporary pupil dilation and blurred vision

Do not use any more frequently than once every 24 hours

What time of day should glycopyrronium tosylate be applied?

There is no recommended time of day for application; make it part of daily routine, for ease of remembering (e.g. before bed each night) [12]

How should glycopyrronium tosylate be stored?

Store at $20-25{ }^{\circ} \mathrm{C}$; excursions to $15-30{ }^{\circ} \mathrm{C}$ permitted

Keep away from (and avoid use near) heat or flame, as flammable

What are the contraindications to the use of glycopyrronium tosylate?

Medical conditions that can be worsened by the anticholinergic effect of glycopyrronium tosylate (e.g. glaucoma, paralytic ileus, unstable cardiovascular status in acute haemorrhage, severe ulcerative colitis, toxic megacolon complicating ulcerative colitis, myasthenia gravis, Sjogren's syndrome)

What other special warnings/precautions pertain to the use of the glycopyrronium tosylate?

Worsening of urinary retention Use with caution in pts with documented urinary retention (or history of such)

Be alert for signs or symptoms of this (e.g. difficulty passing urine, distended bladder), especially in pts with prostatic hypertrophy or bladder-neck obstruction; instruct pts to discontinue immediately and consult a physician if signs or symptoms develop

Control of body temperature With anticholinergic drugs such as glycopyrronium tosylate, heat illness (hyperpyrexia and heat stroke due to decreased sweating) can occur in hot environments

Advise recipients to watch for generalized lack of sweating in very warm or hot environments and to avoid use if they observe this

Operating machinery or an automobile

May cause transient blurred vision; if blurred vision occurs, pts should discontinue use, and refrain from activities that require clear vision (e.g. operating a motor vehicle or other machinery; performing hazardous work), until symptoms resolve

Overdosage Compared with other anticholinergic drugs, symptoms of glycopyrronium overdosage are generally more peripheral than central

Topical overdosage could produce an increase in the incidence and severity of LSRs

Using in occlusive conditions may increase anticholinergic adverse effects (e.g. dry mouth, urinary hesitation)

What clinically relevant drug interactions may potentially occur with glycopyrronium tosylate?

Other anticholinergic-containing Avoid coadministration; may result in increased anticholinergic adverse effects drugs

How should glycopyrronium tosylate be used in special populations?

Pregnant women No data

Breast-feeding women No data; consider benefits of breast-feeding, clinical need for glycopyrronium tosylate, and any potential adverse effects of drug or maternal hyperhidrosis on breast-fed infant

Pediatric pts Safety and effectiveness only established in pts aged $\geq 9$ years

Elderly pts Data insufficient to determine whether response in pts aged $\geq 65$ years is different to that in younger pts

Pts with renal failure Elimination of glycopyrronium severely impaired

LSRs local skin reactions, pts patients 
detection and adequate management of hyperhidrosis can considerably improve quality of life [8]. First-line therapy for primary axillary hyperhidrosis has typically involved topical antiperspirants (commonly containing aluminium salts) $[9,10]$. In patients inadequately controlled by these, approved treatments have been limited to options administered in a clinic (namely botulinum toxin A and microwave thermolysis) [9]. Patients with hyperhidrosis frequently report dissatisfaction with the accessibility, effectiveness and side effects of therapies, further substantiating the need for new effective and well-tolerated treatment options [6].

In the USA, glycopyrronium tosylate cloths (Qbrexza ${ }^{\mathrm{TM}}$ ) have been recently approved for the topical treatment of primary axillary hyperhidrosis in adults and children aged $\geq 9$ years of age. The approval of this new treatment option follows a period of limited innovation in the indication. Table 1 provides a summary of the prescribing information for topical glycopyrronium tosylate as approved in the USA [11]. Consult local prescribing information for further details.

\section{How Does Glycopyrronium Tosylate Work?}

Topical glycopyrronium tosylate acts to reduce the excessive sweat production that characterizes hyperhidrosis by blocking the stimulatory effect of acetylcholine, through competitively inhibiting acetylcholine receptors found on peripheral tissues such as sweat glands $[11,12]$.

\section{What are the Pharmacokinetic Properties of Glycopyrronium?}

When topical glycopyrronium tosylate was used once daily for 5 days in adults and children $\geq 10$ years of age with primary axillary hyperhidrosis, there was no evidence of drug accumulation [11]. Peak plasma concentrations were achieved $1 \mathrm{~h}$ after application in adults and $1.5 \mathrm{~h}$ after application in children aged 10-17 years [11]. Following intravenous administration of glycopyrronium, the mean volume of distribution was $1.3-1.8 \mathrm{~L} / \mathrm{kg}$ in children aged $1-14$ years and $0.42 \mathrm{~L} / \mathrm{kg}$ in adults aged $60-75$ years [11].

While a minor proportion of glycopyrronium is metabolized after intravenous administration, the metabolic pathway has not been characterized [11]. When a radiolabelled intravenous dose of glycopyrronium was given to adults who underwent surgery for gallstones, $\approx 85 \%$ of total radioactivity was recovered in the urine and $<5 \%$ in bile drainage; in both urine and bile, $>80 \%$ of this was unchanged drug [11].

Glycopyrronium pharmacokinetics have not been studied in pregnant women or patients with hepatic impairment [11]. After a $4 \mu \mathrm{g} / \mathrm{kg}$ intravenous dose of glycopyrronium, pharmacokinetic parameters (area under the concentrationtime curve, clearance and 3-h urinary excretion) significantly differed between uremic patients undergoing renal transplantation and healthy patients $(10.6 \mu \mathrm{g} \cdot \mathrm{h} / \mathrm{L}$ vs. $3.73 \mu \mathrm{g} \cdot \mathrm{h} / \mathrm{L}, 0.43$ $\mathrm{L} / \mathrm{h} / \mathrm{kg}$ vs. $1.14 \mathrm{~L} / \mathrm{h} / \mathrm{kg}$ and $0.7 \%$ vs. $50 \%$, respectively). The effects of renal impairment on the pharmacokinetics of topical glycopyrronium tosylate have not been assessed [11].

In vitro studies suggest that clinical use of glycopyrronium tosylate is unlikely to induce cytochrome P450 (CYP) enzymes 1A2, 2B6 and 3A4, or inhibit CYP enzymes 1A2, 2B6, 2C8, 2C9, 2C19, 2D6 and 3A4 [11].

\section{What is the Efficacy of Glycopyrronium Tosylate in Primary Axillary Hyperhidrosis?}

Topical glycopyrronium tosylate was shown to be effective in the treatment of primary axillary hyperhidrosis in two replicate, randomized, double-blind, vehicle-controlled, phase III trials (ATMOS- 1 and ATMOS-2) [13]. ATMOS-1 was conducted in the USA and Germany, while ATMOS-2 was restricted to the USA [13]. The trials enrolled patients $\geq 9$ years of age ( $\geq 18$ years of age in Germany) with:

- primary axillary hyperhidrosis ( $\geq 6$ months' duration);

- sweat production of $\geq 50 \mathrm{mg}$ within 5 minutes in each armpit in at least one of up to three gravimetric measurements taken during the 35-day pre-randomization period;

- an Axillary Sweating Daily Diary (ASDD) or ASDDChildren (ASDD-C; used in patients $<16$ years of age) severity of sweating score $\geq 4$ (measured on an 11-point scale; higher scores indicate greater severity); and

- Hyperhidrosis Disease Severity Scale (HDSS) grade 3 or 4.

Patients received glycopyrronium tosylate or matching vehicle, which they applied once daily to both armpits for 4 weeks [13]. Those who completed ATMOS-1 or ATMOS-2 were eligible to receive up to 44 weeks of openlabel treatment with glycopyrronium tosylate in the ARIDO extension study [14].

At double-blind baseline, patient demographics were generally similar across treatment groups in each trial and patients had a mean age of 32-34 years [13]. With respect to baseline disease characteristics, mean sweat production was $162-183 \mathrm{mg} / 5 \mathrm{~min}$ and mean ASDD/ASDD-C severity of sweating scores were 7.1-7.3. Mean Dermatology Life Quality Index (DLQI) scores were 10.1-12.1 and children's DLQI (CDLQI) scores were 6.9-10.6, indicating that hyperhidrosis had a moderate to very large effect on the lives of 
Table 2 Efficacy of topical glycopyrronium tosylate in primary axillary hyperhidrosis in randomized, double-blind phase III trials

Trial $^{\mathrm{a}}$

Absolute CfB in sweat production $^{\mathrm{b}, \mathrm{c}}(\mathrm{mg} / 5 \mathrm{~min})$
ASDD/ASDD-C HDSS

severity of

sweating

Mean Median LSM $\quad \mathrm{RR}^{\mathrm{b}, \mathrm{d}}(\%$ of pts $) \quad \mathrm{RR}^{\mathrm{e}}\left(\%\right.$ of pts) $\mathrm{RR}^{\mathrm{f}}(\%$ of pts)

Sweat production

\begin{tabular}{|c|c|c|c|c|c|c|}
\hline & \\
\hline & Mean & Median & LSM & $\mathrm{RR}^{\mathrm{b}, \mathrm{d}}(\%$ of pts) & $\mathrm{RR}^{\mathrm{e}}(\%$ of pts $)$ & $\mathrm{RR}^{\mathrm{f}}(\%$ of pts) \\
\hline \multicolumn{7}{|l|}{ ATMOS-1 [13] } \\
\hline Glycopyrronium tosylate once-daily $(n=229)$ & -104.9 & -80.8 & -102.0 & $52.8^{* *}$ & $56.5^{* *}$ & $72.4 * *$ \\
\hline Vehicle $(n=115)$ & -91.9 & -65.8 & -100.3 & 28.3 & 23.7 & 53.2 \\
\hline Glycopyrronium tosylate once-daily $\left[\mathrm{psa}^{\mathrm{g}}\right](n=220)$ & -96.2 & -82.0 & $-100.6^{*}$ & & & \\
\hline Vehicle $\left[\mathrm{psa}^{\mathrm{g}}\right](n=110)$ & -90.6 & -65.1 & -88.1 & & & \\
\hline \multicolumn{7}{|l|}{ ATMOS-2 [13] } \\
\hline Glycopyrronium tosylate once-daily $(n=234)$ & -110.3 & -78.8 & $-115.4 * *$ & $66.1 * *$ & $61.6 * *$ & $77.3^{* *}$ \\
\hline Vehicle $(n=119)$ & -92.2 & -57.9 & -81.2 & 26.9 & 27.8 & 53.3 \\
\hline \multicolumn{7}{|l|}{ Pooled analysis of ATMOS-1 and ATMOS-2 [13] } \\
\hline Glycopyrronium tosylate once-daily $(n=463)$ & -107.6 & -79.8 & $-108.8^{* *}$ & $59.5^{* *}$ & $59.1 * *$ & $74.9 * *$ \\
\hline Vehicle $(n=234)$ & -92.1 & -61.8 & -90.6 & 27.6 & 25.7 & 53.2 \\
\hline \multicolumn{7}{|c|}{ Pediatric pts aged 9 to $\leq 16$ years (pooled data from ATMOS-1 and ATMOS-2) ${ }^{\mathrm{h}}[16]$} \\
\hline Glycopyrronium tosylate once-daily $(n=25)$ & -67.9 & -64.2 & & 59.9 & 61.3 & 79.9 \\
\hline Vehicle $(n=19)$ & -77.8 & -53.7 & & 13.0 & 20.3 & 54.8 \\
\hline \multicolumn{7}{|c|}{ Pts aged $>16$ years (pooled data from ATMOS-1 and ATMOS-2) ${ }^{\mathrm{h}}[16]$} \\
\hline Glycopyrronium tosylate once-daily $(n=438)$ & -109.9 & -80.6 & & 60.2 & 58.7 & 74.3 \\
\hline Vehicle $(n=215)$ & -93.3 & -62.0 & & 28.8 & 26.0 & 53.0 \\
\hline
\end{tabular}

$A S D D(-C)$ Axillary Sweating Daily Diary (-Children; used in pts $<16$ years old), $B L$ baseline, $C f B$ change from baseline, $H D S S$ Hyperhidrosis Disease Severity Scale, ITT intent-to-treat, $L S M$ least squares mean, $p s a$ pre-specified sensitivity analysis, $p t s$ patients, $R R$ responder rate

$* p=0.001, * * p<0.001$ vs. vehicle ( $p$-values for pooled analyses are nominal only)

${ }^{a}$ Analyses conducted in the ITT population at week 4

${ }^{\mathrm{b}}$ Co-primary endpoint in ATMOS-1 and ATMOS-2

${ }^{\mathrm{c}}$ Gravimetrically measured under controlled conditions (average of both axillae)

${ }^{\mathrm{d}}$ Proportion of pts with $\geq 4$-point improvement from BL in weekly average ASDD/ASDD-C severity of sweating responses

${ }^{\mathrm{e}}$ Proportion of pts with $\geq 2$-grade improvement from BL in HDSS

${ }^{\mathrm{f}}$ Proportion of pts with $\geq 50 \%$ reduction from BL in gravimetrically measured sweat production (average of both axillae)

${ }^{g}$ Excluding an analysis centre with extreme outlier data for gravimetrically measured sweat production

${ }^{\text {h}}$ Post hoc comparisons in ATMOS-1 and ATMOS-2; analyses of statistical significance not performed

randomized patients. In each double-blind trial, the majority of randomized patients ( $>90 \%$ ) completed week 4 [13]. Most of these patients entered ARIDO $(87 \% ; n=564 ; 550$ analysed), with $40 \%(n=226)$ of those enrolled completing 44 weeks of open-label treatment [14].

Glycopyrronium tosylate was effective in reducing sweat production in these pivotal trials [13]. Relative to vehicle treatment, glycopyrronium tosylate was associated with a significantly greater reduction in absolute sweat production from baseline to week 4 in ATMOS-2, albeit not in ATMOS-1 (co-primary endpoint; Table 2); however, in a pre-specified sensitivity analysis which identified and excluded one analysis centre in ATMOS-1 with extreme outlier data for gravimetrically measured sweat production, glycopyrronium tosylate was significantly more effective than vehicle in reducing sweat production in the remaining ATMOS-1 patients (Table 2). In both trials, significantly more glycopyrronium tosylate recipients than vehicle recipients had a sweat production response at week 4 (Table 2).

Glycopyrronium tosylate was also effective in reducing patient-reported disease severity [13]. In both trials, ASDD/ ASDD-C severity of sweating responder rates were significantly higher in glycopyrronium tosylate recipients than vehicle recipients at week 4 (co-primary endpoint; Table 2). Across trials, the proportion of glycopyrronium tosylate recipients with an ASDD/ASDD-C severity of sweating response was approximately twice that of vehicle recipients (59.5\% vs. $27.6 \%$; Table 2). Treatment groups diverged with respect to ASDD/ASDD-C severity of sweating responder rates as early as week 1 in both trials [13]. HDSS responses also occurred in significantly higher proportions of glycopyrronium tosylate recipients than vehicle recipients at week 4 of ATMOS-1 and ATMOS-2 (Table 2). 
Glycopyrronium tosylate reduced the disease burden of primary axillary hyperhidrosis relative to vehicle treatment, as indicated by other patient-reported outcomes (PROs) for which significance testing was performed [15]. Quality of life was significantly improved from baseline to week 4 with glycopyrronium tosylate relative to vehicle, as measured by the DLQI in patients $>16$ years of age $(-8.1$ vs. -4.3 in ATMOS- 1 and -8.6 vs. -5.0 in ATMOS- $2 ; p<0.001$ in each trial) and the CDLQI in patients $\leq 16$ years of age ( -7.5 vs. -1.6 and -8.4 vs. $-2.1 ; p<0.05$ in ATMOS 2 ). Changes in Patient Global Impression of Change were similarly more favourable with glycopyrronium tosylate than vehicle ( $p<0.01$ in each trial) [15].

Glycopyrronium tosylate continued to effectively treat primary axillary hyperhidrosis throughout the longerterm ARIDO extension study [14]. At week 44 [or end of treatment/early termination (ET)], gravimetrically measured median sweat production had decreased by $71.3 \%$ from the double-blind baseline (median sweat production $35.7 \mathrm{mg} / 5 \mathrm{~min}$ at week $44 / \mathrm{ET}$ vs. $116.9 \mathrm{mg} / 5 \mathrm{~min}$ at double-blind baseline and $32.8 \mathrm{mg} / 5 \mathrm{~min}$ after 4 weeks of double-blind treatment). At week 44/ET, the HDSS response rate was $63 \%$ (vs. $59 \%$ after 4 weeks of double-blind treatment with glycopyrronium tosylate); $31 \%, 47 \%$ and $17 \%$ of patients had improved from double-blind baseline by 1,2 and 3 grades, respectively (vs. $32 \%, 44 \%$ and $14 \%$ of glycopyrronium tosylate recipients after 4 weeks of double-blind treatment). With respect to quality of life, improvements in DLQI and CDLQI were also largely maintained at week 44/ ET (mean changes from baseline of -8.7 and -6.2 , vs. -8.4 and -8.1 after 4 weeks of double-blind treatment) [14].

The efficacy of glycopyrronium tosylate in children aged 9 to $\leq 16$ years of age was generally similar to that seen in older patients ( $>16$ years of age) in subgroup analyses of data from the double-blind trials (Table 2) and ARIDO [14, 17]. At week 4, children showed improvements in ASDD/ ASDD-C severity of sweating response rate (Table 2), sweat production (Table 2), HDSS response rate (Table 2) and quality of life (DLQI/CDLQI) with glycopyrronium tosylate relative to vehicle that were generally consistent with those seen in older patients [16]. As was the case in the overall population, improvements in disease severity, sweat production and quality of life were maintained over 44 weeks of open-label treatment in children [14, 17]. The benefits of glycopyrronium tosylate over vehicle treatment for primary axillary hyperhidrosis were also generally consistent across subgroups based on location of hyperhidrosis [multifocal (glycopyrronium tosylate applied to axillae only) vs. axillary], receipt of prior treatment, gender, race (white vs. nonwhite) and body mass index in ATMOS- 1 and ATMOS-2 (pooled analysis) [18]. (a)

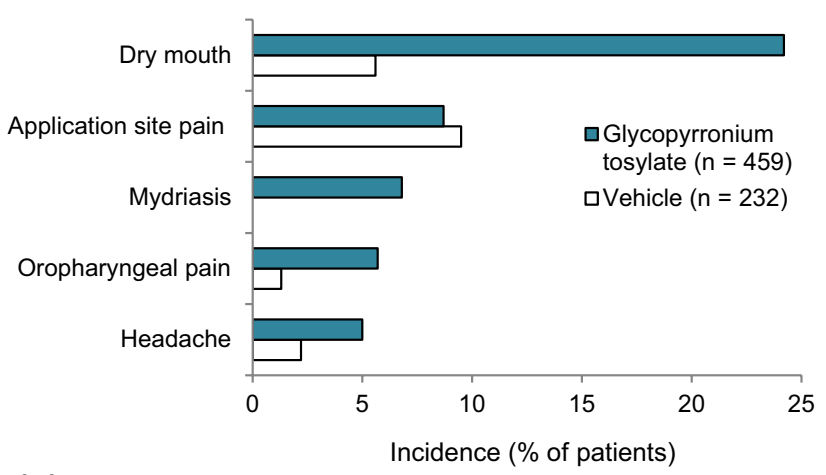

(b)

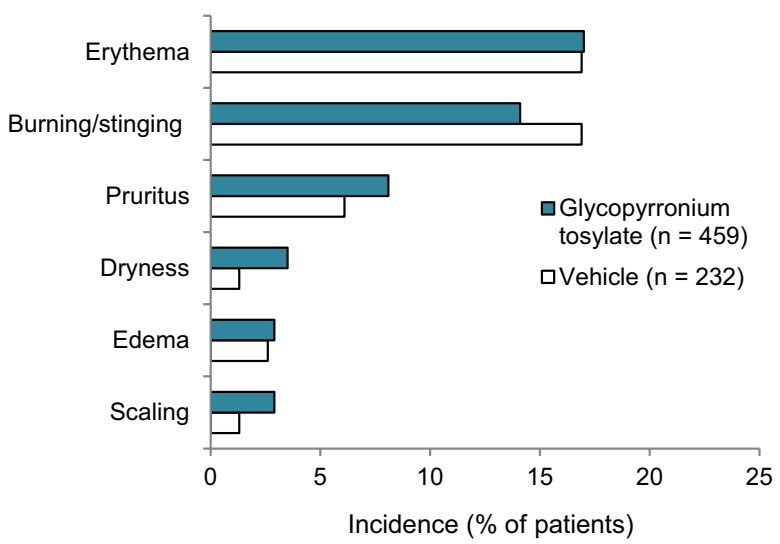

Fig. 1 Incidence of a common treatment-emergent adverse events (occurring in $\geq 5 \%$ of patients in either treatment group) and b local skin reactions, as reported in the ATMOS-1 and ATMOS- 2 trials in primary axillary hyperhidrosis (pooled population) [13]

\section{What is the Tolerability Profile of Glycopyrronium Tosylate?}

Topical glycopyrronium tosylate is generally well tolerated, with most treatment-emergent adverse events (TEAEs) reported in the pivotal ATMOS-1 and ATMOS-2 clinical trials being of mild to moderate severity and transient [13]. In an analysis of pooled data from these 4-week trials, TEAEs were reported in $56.0 \%$ of glycopyrronium tosylate recipients (vs. $32.3 \%$ of vehicle recipients) and drug-related TEAEs were reported in $39.0 \%$ (vs. $16.4 \%$ ); severe TEAEs and serious TEAEs occurred at relatively low rates $(0.9 \%$ vs. $0 \%$ and $0.4 \%$ vs. $0 \%$, respectively). The most common TEAEs were dry mouth, application site pain and mydriasis (Fig. 1). TEAEs infrequently led to treatment discontinuation, doing so in $3.7 \%$ of glycopyrronium recipients and $0.4 \%$ of vehicle recipients. Only one serious TEAE was considered to be related to the study drug (a case of moderate unilateral mydriasis in a glycopyrronium tosylate recipient). Local skin reactions (LSRs) occurred in $30.8 \%$ of glycopyrronium tosylate recipients (versus $30.3 \%$ of vehicle 
recipients) and were most frequently erythema and/or burning/stinging (Fig. 1); LSRs were assessed as severe in $0.2 \%$ (versus $0.9 \%$ ) of patients [13].

As an anticholinergic drug, certain class effects are possible with glycopyrronium tosylate (see Table 1 for warnings and precautions) [11]. Glycopyrronium tosylate is contraindicated in patients with medical conditions that could be worsened by its anticholinergic effect (Table 1) [11]. Aside from dry mouth and mydriasis (Fig. 1), anticholinergic TEAEs occurring in $>2 \%$ of patients in either treatment arm of ATMOS- 1 or ATMOS- 2 were urinary hesitation $(3.5 \%$ with glycopyrronium tosylate vs. $0 \%$ with vehicle in the pooled population), blurred vision (3.5\% vs. $0 \%)$, nasal dryness ( $2.6 \%$ vs. $0.4 \%)$, dry eye $(2.4 \%$ vs. $0.4 \%)$, constipation $(2 \%$ vs. $0 \%)$ and urinary retention (1.5\% vs. $0 \%)$ [13]. In glycopyrronium tosylate recipients, most cases of mydriasis were unilateral (23/31 events), while most cases of blurred vision were bilateral (11/16 events) [13]. There have also been case reports of reversible unilateral mydriasis in patients treated with topical anticholinergics (including glycopyrronium tosylate), likely resulting from accidental ocular exposure $[19,20]$; clinicians should be aware of this potential benign etiology in patients presenting with mydriasis [20].

Longer-term safety data from up to 44 weeks of openlabel treatment in the ARIDO extension study were generally consistent with those observed during double-blind treatment [14]. The most common TEAEs were dry mouth (16.9\% of patients), blurred vision (6.7\%; 40/45 events bilateral), application site pain (6.4\%), nasopharyngitis $(5.8 \%)$, mydriasis $(5.3 \% ; 31 / 37$ events unilateral) and urinary hesitation $(4.2 \%)$. The majority of TEAEs occurred during the first 12 weeks, with frequencies tending to decrease thereafter. TEAEs led to treatment discontinuation in $8 \%$ of patients. Serious TEAEs were reported in seven patients $(1.3 \%)$ and one of these was considered to be related to treatment (mydriasis). LSRs occurred in $32.5 \%$ of patients, with the most frequent being erythema (21.1\%), burning/stinging (13.3\%) and pruritus (12.4\%). Severe LSRs were infrequent (experienced by $3 \%$ of the 550 patients who comprised the safety population and $8 \%$ of the 179 patients who experienced LSRs) and neither the incidence nor the severity of LSRs increased over time. Anticholinergic TEAEs of special interest (i.e. blurred vision, mydriasis and symptoms of urinary retention or hesitation) were generally managed by dose interruption or no action and typically resolved within 3-14 days of onset; only one case was severe (blurred vision) [14].

In a subgroup of children aged between 9 and 16 years, TEAEs reported during ATMOS- 1 or ATMOS-2 $(n=25$ receiving glycopyrronium tosylate) and ARIDO ( $n=28$ [14]) were similar to those in patients aged $>16$ years $[16$, 17]. Children rarely discontinued glycopyrronium tosylate due to TEAEs (one child during double-blind treatment and one during ARIDO) [17]. Severe TEAEs were experienced by one child during double-blind treatment with glycopyrronium tosylate and one child during ARIDO [17]. No serious TEAEs occurred in children [16, 17].

\section{What is the Current Clinical Position of Glycopyrronium Tosylate in Primary Axillary Hyperhidrosis?}

Primary axillary hyperhidrosis is associated with considerable patient burden, with patients reporting embarrassment and detrimental effects on many facets of their lives [2, 5]. The International Hyperhidrosis Society recommends glycopyrronium tosylate as a first-line treatment option for primary axillary hyperhidrosis, alongside topical antiperspirant therapy (containing aluminium and zirconium salts) [9]. Patients administered these treatments should be educated as to the application time and technique, in order to maximise efficacy and minimise adverse effects. Glycopyrronium tosylate is also an option in patients who have had an unsatisfactory response to topical antiperspirant therapy (or experienced intolerable side effects), with the other options in this setting being botulinum toxin A injections and microwave thermolysis [9].

Once-daily use of topical glycopyrronium tosylate reduces disease severity (as assessed using the ASDD/ ASDD-C, a novel reliable and validated tool in the clinical evaluation of treatments for axillary hyperhidrosis [21]) and gravimetrically measured sweat production relative to vehicle treatment in patients with primary axillary hyperhidrosis (Table 1). Importantly, glycopyrronium tosylate also significantly improves quality of life parameters in these patients [13]. Efficacy has been shown to be maintained for up to 48 weeks of treatment [14]; given the chronic nature of hyperhidrosis, real-world studies following patients using glycopyrronium tosylate over longer periods would be of interest. Unlike botulinum toxin A and microwave thermolysis, glycopyrronium tosylate is specifically approved for use in children $\geq 9$ years of age in the USA (Table 1). The efficacy of glycopyrronium tosylate in children is comparable to that seen in older patients $[14,17]$.

Glycopyrronium tosylate is generally well tolerated in both adults and children ( $\geq 9$ years of age), with most reported TEAEs being mild to moderate in severity [13, 14]. While topical administration does limit the systemic anticholinergic effects of glycopyrronium, they are not completely eliminated; the most common TEAEs with glycopyrronium tosylate are systemic or local anticholinergic events (e.g. dry mouth, mydriasis) and reactions at the application site (e.g. erythema, burning/stinging) (Fig. 1). TEAEs are generally transient and become less common as treatment progresses [13, 14]. 
Currently, there are no data comparing glycopyrronium tosylate with other approved treatments for primary axillary hyperhidrosis; such data could help clarify the position of glycopyrronium tosylate in treatment of the disease. Although glycopyrronium tosylate does require daily administration (Table 1), some patients who are not controlled on topical antiperspirant therapy may find glycopyrronium tosylate more convenient than other secondline options, as it can be self-administered outside of a clinic. From a commercial payer perspective, introducing glycopyrronium tosylate to a health plan formulary was found to have a modest budgetary impact, based on the inputs of the primary scenario of a US budget impact analysis [22]. Appropriate management of primary axillary hyperhidrosis may result in cost-offsets, which may reduce the real-world incremental budget impact realized by payers [22].

As the only approved topical anticholinergic treatment for primary axillary hyperhidrosis in the USA, glycopyrronium tosylate offers a self-administered, non-invasive and generally well-tolerated alternative to topical antiperspirant therapy and clinic-based treatment options in adult patients, and is the only alternative to topical antiperspirant therapy to be specifically approved in children and adolescents $\geq 9$ years of age.

Acknowledgements The manuscript was reviewed by: A. Y. Moore, Arlington Center for Dermatology, Arlington Research Center, Arlington, TX, USA; N. Wolosker, Hospital Israelita Albert Einstein, São Paulo, Brazil. During the peer review process, Dermira, the marketingauthorization holder of glycopyrronium tosylate topical formulation, was also offered an opportunity to provide a scientific accuracy review of their data. Changes resulting from comments received were made on the basis of scientific and editorial merit.

\section{Compliance with ethical standards}

Funding The preparation of this review was not supported by any external funding.

Conflict of interest Y N. Lamb is an employee of Adis International Ltd./Springer Nature, is responsible for the article content and declares no conflicts of interest.

Open Access This article is distributed under the terms of the Creative Commons Attribution-NonCommercial 4.0 International License (http://creativecommons.org/licenses/by-nc/4.0/), which permits any noncommercial use, duplication, adaptation, distribution and reproduction in any medium or format, as long as you give appropriate credit original author(s) and the source, provide a link to the Creative Commons licence and indicate if changes were made.

\section{References}

1. Doolittle J, Walker P, Mills T, et al. Hyperhidrosis: an update on prevalence and severity in the United States. Arch Dermatol Res. 2016:308:743-9.
2. Hasimoto EN, Cataneo DC, dos Reis TA, et al. Hyperhidrosis prevalence and its impact on population. Shanghai Chest. 2019;3:30.

3. McConaghy JR, Fosselman D. Hyperhidrosis: management options. Am Fam Physician. 2018;97(11):729-34.

4. Walling HW. Clinical differentiation of primary from secondary hyperhidrosis. J Am Acad Dermatol. 2011;64(4):690-5.

5. Jacob CI. Examining hyperhydrosis: an update on new treatments. Am J Manag Care. 2018;24:S496-501.

6. Kamudoni P, Mueller B, Halford J, et al. The impact of hyperhidrosis on patients' daily life and quality of life: a qualitative investigation. Health Qual Life Outcomes. 2017;15:121.

7. Bahar R, Zhou P, Liu Y, et al. The prevalence of anxiety and depression in patients with or without hyperhidrosis (HH). J Am Acad Dermatol. 2016;75:1126-33.

8. Haider A, Solish N. Focal hyperhidrosis: diagnosis and management. CMAJ. 2005;172(1):69-75.

9. International Hyperhidrosis Society. Primary focal axillary hyperhidrosis. 2018. http://www.sweathelp.org/. Accessed 17 Sep 2019.

10. Grabell DA, Hebert AA. Current and emerging medical therapies for primary hyperhidrosis. Dermatol Ther (Heidelb). 2017;7:25-36.

11. Dermira Inc. Qbrexza (glycopyrronium cloth): US prescribing information. 2018. http://pi.dermira.com/. Accessed 17 Sep 2019.

12. Dermira Inc. Qbrexza: frequently asked questions. 2019. http:// www.qbrexza.com/. Accessed 17 Sep 2019.

13. Glaser DA, Hebert AA, Nast A, et al. Topical glycopyrronium tosylate for the treatment of primary axillary hyperhidrosis: results from the ATMOS- 1 and ATMOS-2 phase 3 randomized controlled trials. J Am Acad Dermatol. 2019;80(1):128-38.e2.

14. Glaser DA, Hebert AA, Nast A, et al. A 44-week open-label study evaluating safety and efficacy of topical glycopyrronium tosylate in patients with primary axillary hyperhidrosis. Am J Clin Dermatol. 2019;20(4):593-604.

15. Pariser DM, Hebert AA, Drew J, et al. Topical glycopyrronium tosylate for the treatment of primary axillary hyperhidrosis: patient-reported outcomes from the ATMOS-1 and ATMOS-2 phase III randomized controlled trials. Am J Clin Dermatol. 2019;20(1):135-45.

16. Hebert AA, Glaser DA, Green L, et al. Glycopyrronium tosylate in pediatric primary axillary hyperhidrosis: post hoc analysis of efficacy and safety findings by age from two phase three randomized controlled trials. Pediatr Dermatol. 2019;36(1):89-99.

17. Hebert AA, Glaser DA, Green L, et al. Short- and long-term efficacy and safety of topical glycopyrronium cloth for the treatment of primary axillary hyperhidrosis: post hoc pediatric subgroup analyses from the phase 3 studies [poster no. S68]. Skin. 2018;2(Suppl 2):S68.

18. Glaser DA, Green L, Drew J, et al. Glycopyrronium cloth improves axillary hyperhidrosis across a broad spectrum of patients: post hoc analyses of the ATMOS- 1 and ATMOS- 2 phase 3 randomized controlled trials in patient subpopulations [abstract no. 8599]. In: 77th Annual Meeting of the American Academy of Dermatology. 2019.

19. Micieli R, Micieli JA. Dilated pupil in a patient with hyperhidrosis. JAMA. 2019;322(3):264-5.

20. Pashaei-Marandi A, Assam JH, Arnold A, et al. Reversible anisocoria due to inadvertent ocular exposure to topical anticholinergic treatment for primary axillary hyperhidrosis. Can J Ophthalmol. 2019. https://doi.org/10.1016/j.jcjo.2019.04.009.

21. Nelson LM, DiBenedetti D, Pariser DM, et al. Development and validation of the Axillary Sweating Daily Diary: a patientreported outcome measure to assess axillary sweating severity. J Patient Rep Outcomes. 2019;3:59.

22. Gillard K, Moradi A, Bloudek L. Budget impact analysis of glycopyrronium cloth for the treatment of primary axillary hyperhidrosis [abstract no. L14]. J Manag Care Spec Pharm. 2018;24(Suppl 10-a):S79 